\title{
FUNGOS FILAMENTOSOS DO SOLO DA ILHA DOS EUCALIPTOS NA REPRESA DO GUARAPIRANGA EM SÃO PAULO, SP'
}

\author{
Valéria Barros dos Santos 2 \\ Christian Wellbaum ${ }_{2}^{2}$ \\ Iracema Helena Schoenlein-Crusius ${ }^{2}$
}

\section{Recebido em 2/9/97. Aceito em 14/4/98}

\begin{abstract}
RESUMO - (Fungos filamentosos do solo da Ilha dos Eucaliptos na Represa do Guarapiranga em São Paulo, SP). Foi estudada a diversidade dos fungos do solo da Itha dos Eucaliptos na Represa do Guarapiranga em São Paulo, SP, sob floresta de Eucalyptus, mata de interseção e mata secundária remanescente, em duas épocas climaticamente distintas: março - época quente e úmida (verão) e junho - época fria e seca (inverno) de 1996. Os fungos filamentosos foram isolados pelos métodos da diluição $\mathrm{em}$ série e placa de solo. A similaridade entre as micotas foi estabelecida pelo indice de Sörensen. No total foram obtidos 44 táxons de fungos (Zygomycota, Ascomycota e fungos mitospóricos), distribuidos em 92 ocorrências. No solo sob a floresta de Eucalyptus spp. foram encontrados 20 táxons ( 31 ocorrências) de fungos, no solo da interseção 32 táxons ( 38 ocorrências) e no solo da mata secundária 16 táxons ( 23 ocorrências). Similaridade de $47 \%$ foi encontrada entre a micota do solo sob Eucalyptus com a da interseção, e desta com a micota da mata secundária. A micota do solo sob Eucalyptus e a da mata secundária apresentaram $39 \%$ de similaridade. Na época quente e úmida (verão) a micota terrestre da Ilha foi composta por 26 táxons de fungos ( 47 ocorrências) e na época fria e seca (inverno) por 32 táxons ( 45 ocorrèncias), com $46 \%$ de similaridade entre ambas. Pela diluição em série foram isolados 33 táxons ( 60 ocorrências) e pela técnica da placa de solo, 22 táxons (32 ocorrências). $\mathrm{O}$ número de colônias de fungos foi significativamente maior no solo da mata secundária, seguido pela vegetação de interseção e a floresta de Eucalyptus, independentemente da época de coleta. O possivel efeito inibitório de Eucalyptus sobre a comunidade dos fungos do solo da Ilha pode ter sido expresso pela diminuição do número de colônias na área reflorestada, no entanto, não afetou a diversidade dos fungos, que se apresentou elevada nos três tipos de vegetação.
\end{abstract}

Palavras-chaves: fungos terrestres, vegetação, Eucalyptus, sistema lêntico

ABSTRACT - (Filamentous fungi from the soil of the "Ilha dos Eucaliptos" in the "Represa do Guarapiranga" in São Paulo State, SP). The aim of the present study was to compare the diversity of the fungi in the soil of the "Illha dos Eucaliptos", located in the dam "Represa do Guarapiranga", in São Paulo, SP, under an Eucalyptus, intermediate and a remaining secundary forest, in March - warm and moist season (summer) and June - cold and dry season (winter) of 1996. The filamentous soil fungi were isolated by the dilution plate and soil plate methods. The similarity among the mycotas was

\footnotetext{
'Parte do trabalho de conclusào do curso de Bacharelado em Ciências Biológicas na Universidade de Santo Amaro (UNISA), São Paulo, SP da primeira autora. Auxilio FAPESP

Instituto de Botânica, C. Postal 4005, CEP 01061-970, São Paulo, SP, Brasil
} 
stablished by the index of Sörensen. A total number of 44 fungal taxa (Zygomycota, Ascomycota and mitosporic fungi), distributed in 92 registers was obtained. Twenty fungal taxa ( 31 registers) were obtained from the Eucalyptus spp. forest, 32 taxa (38 registers) from the intermediate and 16 taxa (32 registers) from the secundary forest. A similarity of $47 \%$ was found comparing the soil mycota under Eucalyptus and the intermediate forest, as well as comparing the latter with the mycota of the secundary forest. The similarity between the mycota of the Eucalyptus and the secondary forest was of $39 \%$. In summer the terrestrial mycota was composed by 26 taxa ( 47 registers) and in the winter by 32 taxa ( 45 registers), with $46 \%$ of similarity between them. The dilution plate method allowed the isolation of 33 fungal taxa ( 60 registers) and the soil plates 22 taxa ( 32 registers). Total number of colonies was significantly higher in the soil of the secondary forest, followed by the intermediate and Eucalyptus forest regardless of the climatic conditions. A possible inhibitory effect of the Eucalyptus, forest on the terrestrial fungal community of the island may have been expressed by the decrease of the number of fungal colonies in the soil of the forested site, although the fungal diversity was not affected, remaining high at the three types of vegetation.

Key words: terrestrial fungi, vegetation, Eucalyptus, lentic systems

\section{Introdução}

Considera-se que a diversidade dos fungos terrestres pode estar estreitamente associada ao tipo de vegetação e aos fatores climáticos dos ecossistemas (Dix \& Webster 1995). As modificações da vegetação causadas pela impacto ambiental como queimadas, desmatamento ou reflorestamento podem refletir-se sobre a diversidade da micota terrestre e, consequentemente, sobre os mecanismos relacionados com a manutenção da fertilidade do solo (Christensen 1989).

O reflorestamento tem sido assunto polêmico sob o ponto de vista ecológico, principalmente no caso de espécies arbóreas exóticas, como Pinus spp. e Eucalyptus spp., conhecidas produtoras de substâncias alelopáticas. Em estudos sobre os fungos isolados do solo sob floresta tropical úmida implantada com diferentes talhões de Pinus sp., Ceccato et al. (1986) verificaram que os efeitos alelopáticos dependiam da espécie arbórea implantada e do grupo de fungos estudados (basidiomicetos, fungos filamentosos terrestres e micorrizicos).

À presença de Eucalyptus têm sido associadas alterações de nascentes e do lençol freático, ressecamento, empobrecimento e conseqüente esterilização do solo, efeitos alelopáticos sobre outras formas de vegetação e extinção da fauna (Lima 1993). Por outro lado, a presença de Eucalyptus nos ecossistemas também tem sido considerada benéfica para a recuperação da estrutura do solo, devido à deposição de material orgânico (Salomão 1993), proteção com espessa camada de folhas e detritos e diminuição do perigo de erosão (Poggiani 1995).

Della Bruna et al. (1989) verificaram que a presença de extrato de serapilheira de talhões de Eucalyptus refletiu-se sobre a composição das espécies de fungos, porém não sobre o número de colônias. Maior atividade da microbiota no solo sob mata nativa do que sob floresta de Eucalyptus tem sido atribuida, em parte, ao acúmulo de material orgânico não decomposto e à presença de componentes com atividade antibacteriana (Della Bruna et al. 1991)

Em termos de diversidade dos fungos associados ao Eucalyptus no Brasil são conhecidos alguns táxons potencialmente fitopatógenos (Crous et al. 1989) e 
decompositores de folhedo (Sutton \& Hodges 1975; 1976), havendo somente informações esparsas sobre a diversidade dos fungos do solo sob as áreas reflorestadas.

Há mais de vinte anos, aproximadamente $70 \%$ da Ilha dos Eucaliptos, situada na Represa do Guarapiranga na cidade de São Paulo, foi reflorestada com diversas espécies de Eucalyptus, havendo ainda áreas cobertas por mata secundária, semelhante à mata atlântica de planalto (Begovacz 1995). A existência de fisionomias tão distintas no mesmo local tornou a Ilha sítio de estudos adequado para avaliação dos efeitos da vegetação sobre as características microbianas do solo. O objetivo do presente estudo é a comparação da diversidade dos fungos filamentosos do solo sob três tipos de vegetação em duas épocas sazonais distintas, na Ilha dos Eucaliptos, na Represa do Guarapiranga, em São Paulo, SP.

\section{Material e métodos}

A Ilha dos Eucaliptos localiza-se centralmente na Represa do Guarapiranga, a $737 \mathrm{~m}$ de altitude (Rocha 1976), apresentando relevos de curvas suaves a leste e topografia mais acidentada a oeste, atingindo $796 \mathrm{~m}$ de altitude na porção centronorte (Emplasa 1981). Abrange área de $323.500 \mathrm{~m}^{2}$ apresentando vegetação constituída principalmente por reflorestamento de Eucalyptus spp., mata secundária preservada e região de interseção entre entre essas últimas (Silva 1992 apud Begovacz 1995).

O local apresenta terrenos de idade terciária com solo resultante da decomposição de rochas cristalinas, como o gnaisse, a mica-xista e magmáticas como o granito (Almeida 1981). As suas características morfológicas causam os processos erosivos que ocorrem nas margens da ilha (Begovacz 1995).

$\mathrm{Na}$ floresta de Eucalyptus, na mata secundária e na região de interseção foram estabelecidos cinco pontos para as coletas ( $300 \mathrm{~g}$ de solo até $15 \mathrm{~cm}$ de profundidade), que foram efetuadas no dia 06 de março (verão) e 04 de junho de 1996 (inverno), entre $10 \mathrm{e} 11 \mathrm{~h}$ da manhã. Os dados de precipitação (chuvas) dos meses e dos dias em que foram conduzidas as coletas foram obtidos na Estação Meteorológica do Instituto Geofísico e Astronômico da Universidade de São Paulo. Concomitantemente, foi determinada a temperatura do solo a $15 \mathrm{~cm}$ de profundidade, com auxílio de termômetro de haste metálica. $\mathrm{O}$ pH do solo coletado foi determinado por leitura em potenciômetro a partir de suspensão de solo em solução aquosa na proporção 1:2,5 (Raij \& Zullo 1977). Para a determinação do teor de umidade do solo, 15 a $20 \mathrm{~g}$ de solo foram colocadas em latas de metal previamente taradas, submetidas a temperatura de $105^{\circ} \mathrm{C}$ até peso constante. $\mathrm{O}$ teor de umidade foi calculado com base no valor percentual hídrico sobre o peso seco (Mc Lean \& Cook 1968).

Para o isolamento dos fungos foram utilizadas as técnicas de placa de solo (Warcup 1950, modificada pela diluição 1:100ml da suspensão do solo) e de diluição em série (Clark 1965), com plaqueamento em meio de Martin (Martin 1961) com rosa de bengala e sulfato de estreptomicina.

Em ambas as técnicas as placas de Petri foram incubadas à temperatura ambiente $\left(20\right.$ a $\left.25^{\circ} \mathrm{C}\right)$ durante cinco dias para quantificação, isolamento e identificação dos fungos filamentosos. 
Após a contagem de colônias, as mesmas foram purificadas em meio de batatadextrose-ágar (BDA) ou "Synthetic-Mucor-agar" - SMA (Difco Manual 1972), para então serem observadas as características morfológicas, tais como coloração (verso e reverso da colônia), diâmetro, textura e presença de exsudatos. As características morfológicas das estruturas somáticas de reprodução (esporos, conídios, conidióforos, etc.) foram analisadas por meio da observação de lâminas (montadas com água e azul de algodão com lactofenol) ao microscópio óptico. Para a identificação dos táxons aos níveis genéricos e específicos foi utilizada literatura pertinente (Barron 1968; Riffai 1969; Barnett \& Hunter 1972; Pitt 1979; Domsch et al. 1980).

Os resultados da ocorrência dos táxons isolados foram submetidos ao teste de similaridade de Sörensen (Brown \& Zar 1984), com a finalidade de comparar a diversidade dos fungos do solo das três florestas estudadas (Eucalyptus, mata secundária e interseção), nas duas coletas (quante e úmida, e fria e seca) e obtidos pelas duas técnicas de isolamento (diluição em série e placa de solo).

Para comparar as médias de temperatura, $\mathrm{pH}$ e teor de umidade do solo e também do número de colônias pelo método da placa de solo entre as três áreas estudadas, foi realizada a análise de variância ANOVA (Brown \& Zar 1984).

\section{Resultados e discussão}

As informações obtidas na Estação Meteorológica do Instituto Astronômico e Geofísico (USP), confirmam que as coletas foram de fato, realizadas em épocas climáticas distintas. A precipitação pluviométrica foi de $18,6 \mathrm{~mm}$ no dia 06 de março (verão) e de zero mm no dia 04 de junho (inverno). No mês de março ocorreu um total de $343,7 \mathrm{~mm}$ e no mês de junho $50,0 \mathrm{~mm}$ de precipitação.

A Tab. 1 apresenta os valores individuais, as médias e os desvios padrão dos parâmetros abióticos determinados no solo durante as coletas. De acordo com a análise de variância, somente as médias do $\mathrm{pH}$ do solo sob vegetação de Eucalyptus spp. são significativamente maiores do que as do solo da área de interseção e da mata secundária, na época quente e úmida $(\mathrm{F}=3,59<5 \%)$ e na época fria e seca $(\mathrm{F}=7,124,>5 \%)$. De acordo com a literatura (Dix \& Webster 1995), os fungos filamentosos terrestres suportam condições extremas de temperatura, $\mathrm{pH}$ e umidade mais acentuadas do que as da Ilha dos Eucaliptos. Os valores da temperatura a $15 \mathrm{~cm}$ de profundidade e do $\mathrm{pH}$ (Tab. 1) têm sido semelhantes, mas os do teor de umidade têm sido menores do que os encontrados no solo da mata atlântica, usualmente maiores do que 50\% (Attili 1994; Ninomiya et al. 1993; Schoenlein-Crusius 1993), caracterizando o solo da Ilha dos Eucaliptos como acentuadamente seco.

Foram encontrados 44 táxons de fungos, distribuídos em 32 espécies de fungos mitospóricos, nove de Zygomycota, dois de Ascomycota e uma levedura (Tab. 2). No Parque Estadual das Fontes do Ipiranga, SP, foram obtidos 39 táxons de fungos do solo, em coletas mensais realizadas de outubro/90 a março/91 (Ninomiya et al. 1993) e na Reserva Biológica do Alto da Serra, SP, foram isolados 32 táxons, em coletas realizadas mensalmente, de outubro/90 a março/91 (Antunes et al. 1993). Portanto, o número de táxons aqui encontrado pode ser considerado elevado quando comparado 
Tabela 1. Médias ( $\mathrm{n}=5$ ) e desvios padrões dos parâmetros abióticos medidos no solo na Ilha dos Eucaliptos, Represa do Guarapiranga, São Paulo, SP.

\begin{tabular}{|c|c|c|c|c|c|c|c|c|c|}
\hline \multicolumn{10}{|c|}{ Verão (06/ março/ 1996) } \\
\hline \multirow[t]{2}{*}{ Pontos } & \multicolumn{3}{|c|}{ temperatura ${ }^{\circ} \mathrm{C}$} & \multicolumn{3}{|c|}{$\mathrm{pH}$} & \multicolumn{3}{|c|}{ umidade $\%$} \\
\hline & Eucalipto & Interseçào & Mata & Eucalipto & Interseção & Mata & Eucalipto & Interseção & Mata \\
\hline 1 & 21,0 & 20,0 & 19,0 & 5,1 & 4,8 & 4,5 & 26,30 & 57,00 & 34,06 \\
\hline 2 & 20,0 & 19,0 & 19,0 & 4,9 & 5,0 & 4,7 & 33,39 & 34,90 & 30,41 \\
\hline 3 & 22,0 & 20,0 & 20,0 & 5,0 & 4,9 & 5,0 & 28,26 & 46,16 & 30,83 \\
\hline 4 & 19,5 & 19,0 & 20,0 & 5,2 & 4,7 & 4,8 & 27,08 & 26,19 & 30,91 \\
\hline 5 & 19,0 & 18,0 & 20,0 & 4,8 & 4,6 & 4,6 & 38,56 & 32,88 & 32,11 \\
\hline Médias & 20,3 & 19,2 & 19,6 & 5,0 & 4,8 & 4,7 & 30,71 & 39,42 & 31,66 \\
\hline \multirow[t]{2}{*}{ Desvios } & 1,07 & 0,74 & $0,48-$ & 0,14 & 0,14 & 0,17 & $4,63-$ & 10,88 & 1,32 \\
\hline & \multicolumn{9}{|c|}{ Inverno (04/ junho/ 1996) } \\
\hline \multirow{2}{*}{ Pontos } & & temperatura ${ }^{\circ} \mathrm{C}$ & & & $\mathrm{pH}$ & & & umidade $\%$ & \\
\hline & Eucalipto & Interseção & Mata & Eucalipto & Interseção & Mata & Eucalipto & Interseção & Mata \\
\hline 1 & 17,0 & 17,0 & 17,0 & 4,4 & 4,0 & 3,7 & 24,79 & 27,08 & 27,97 \\
\hline 2 & 17,0 & 17,0 & 17,0 & 4,5 & 3,8 & 4,1 & 23,04 & 34,25 & 63,65 \\
\hline 3 & 17,0 & 17,0 & 17,0 & 4,5 & 3,7 & 4,3 & 25,71 & 31,31 & 26,69 \\
\hline 4 & 17,0 & 17,0 & 17,0 & 4,4 & 4,4 & 4,1 & 31,14 & 23,90 & 37,35 \\
\hline 5 & 17,0 & 17,0 & 17,0 & 4,4 & 4,1 & 3,9 & 25,35 & 29,16 & 45,73 \\
\hline Médias & 17,0 & 17,0 & 17,0 & 4,4 & 4,0 & 4,0 & 26,00 & 29,14 & 40,27 \\
\hline Desvios & 0 & 0 & 0 & 0,10 & 0,22 & 0,18 & 0,10 & 0,22 & 0,18 \\
\hline
\end{tabular}

aos estudos anteriormente citados, ambos realizados em áreas de mata secundária e de Mata Atlântica, respectivamente.

Os fungos mitospóricos, que geralmente são predominantes no solo, apresentam capacidade sapróbia elevada, alta produção enzimática, esporulação abundante e sistemas de dispersão eficientes, além da capacidade de rápida colonização dos substratos orgânicos no solo (Garrett 1963), caracteristicas que lhes conferem a ubiquidade nos ecossistemas terrestres (Dix \& Webster 1995).

No solo sob os três tipos de vegetação predominaram espécies de Penicillium e Trichoderma, fato que pode ser considerado comum, pois os representantes destes gêneros são extremamente bem adaptados às mais variadas condições do solo (Riffai 1969; Pitt 1979), sendo freqüentemente citados nos levantamentos de fungos de ambientes terrestres na Mata Atlântica (Ninomiya et al. 1993; Antunes et al. 1993; Schoenlein-Crusius 1993; Attili 1994; Garlipp 1995).

Comparando-se a micota isolada na época quente e úmida, quando foram identificados 26 táxons (47 registros), com a da época fria e seca, quando foram isolados 32 táxons (45 registros), obteve-se o indice de similaridade de Sörensen de $46 \%$. A escolha de periodos de estações distintas para as coletas contribuiu para elevar a diversidade de táxons identificados. As variações sazonais interferem na quantidade de propágulos, como também nas populações (Bettuci et al. 1990). Attili (1994), estudando a micota do solo na Mata Atlântica, registrou espécies que cresceram unicamente em um periodo. No presente estudo, o número total de táxons não se 
alterou acentuadamente de uma época para outra, porém a composição das micotas apresentou-se diferente, com espécies isoladas exclusivamente em determinada ocasião (Tab. 2).

Comparando-se os resultados encontrados nas três áreas, verifica-se que a técnica de diluição em série resultou na obtenção de 33 táxons ( 60 ocorrências) e na técnica da placa de solo, o total de 22 táxons de fungos (32 ocorrências). A utilização da técnica de diluição apresentou melhor resultado do que da placa de solo, pois na última, as colônias de Mucorales cresceram demasiadamente sobre as demais, dificultando o isolamento e a purificação das culturas.

No solo sob Eucalyptus spp. foram encontrados 20 táxons ( 31 ocorrências) de fungos, no solo da área de interseção 32 táxons (38 ocorrências) e 16 táxons (23 ocorrências) no solo sob mata secundária. Absidia sp., Aspergillus glaucus, Epicoccum nigrum, Penicillium implicatum, P. montanense, P. verrucosum, Verticillium lecanii foram isolados exclusivamente no solo sob Eucalyptus. Foram observados exclusivamente no solo da área de interseção: Circinella sp., Acremonium sp. Fusarium solani, Penicillium fellutanum, P. furcatum, P. oxalicum, P. purpurogenum, $P$. simplicissimum, $P$. spinulosum, Rhizopus oryzae e Cunninghamella elegans, enquanto no solo sob mata secundária ocorreram exclusivamente Backusella lamprospora, Trichoderma aureoviride, T. viride e Pestalotiopsis sp.

A maioria dos táxons identificados no presente estudo têm sido considerados cosmopolitas, ocorrendo frequentemente em outros ecossistemas, como por exemplo, na Mata Atlântica de Paranapiacaba (Schoenlein-Crusius 1993). No entanto, observase que há elevada incidência das espécies de Penicillium no solo sob os três tipos de vegetação. Possíveis parâmetros que contribuem para esse resultado ainda continuam sendo investigados.

O índice de similaridade de Sörensen foi calculado a partir da soma das ocorrências totais dos fungos no solo das três áreas. O índice correspondeu a $47 \%$ entre a micota do solo sob a floresta de Eucalyptus e a micota da área de interseção, novamente $47 \%$ entre a micota do solo sob mata secundária e a micota do solo da área de interseção e 39\% entre a micota do solo sob Eucalyptus e a micota do solo sob mata secundária, indicando que a diversidade dos fungos na floresta implantada difere acentuadamente da encontrada na mata secundária. A área de interseção possivelmente abrange algumas características da mata secundária e também da floresta implantada, constituindo ambiente favorável para o desenvolvimento da micota terrestre, o que se expressa pelo maior número total e exclusivo de táxons, bem como a mesma similardade entre a micota desta área, com as outras, isto é, mata secundária e Eucalyptus.

Comparando-se os resultados da utilização da técnica da placa de solo com a diluição em série (Tab. 3), verificou-se que os maiores números de colônias de fungos foram registrados no solo da mata secundária, seguido pela interseção e da floresta de Eucalyptus. As diferenças entre os números totais de colônias de fungos nos três tipos de solo apresentaram-se estatisticamente significativas na época quente e úmida $(\mathrm{F}=12$, $>5 \%)$ e na época fria e seca $(\mathrm{F}=4,18>5 \%)$.

Os resultados encontrados sobre os microrganismos no solo da Ilha dos Eucaliptos são contrários aos de Della Bruna et al. (1989) que, trabalhando em talhões, não 
Tabela 2. Número de ocorrências (+ presença/ - ausência) dos fungos do solo na llha dos Eucaliptos, Represa do Guarapiranga, São Paulo, SP, isolados no verão (V) e no inverno (I) pelos métodos de diluição e placa de solo.

\begin{tabular}{lll|cc|cc}
\hline Tipo de vegetação & Eucalyptus spp. & \multicolumn{2}{c|}{ Intersecção } & \multicolumn{2}{c}{ Mata total } \\
Técnica de isolamento & Diluição Placa & Diluição Placa & Diluição Placa
\end{tabular} Técnica de isolamento Época de coleta

Zygomycota Absidia sp.

Backusella lamprospora

Lend \& Hesselt.

Circinella sp.

Cunninghamella sp.

$C$ elegans Lend.

C. phaeospora Lend.

Mucor hiemalis $\mathrm{f}$. hiemalis

Wehmer

Rhizopus oryzae Ehrenb.

R. stolonifer

(Ehrenb. ex Link.) Lind.

Fungos mitospóricos

Acremonium sp.

Aspergillus glaucus

(Mich. ex. L.:Fr) Link.

A. niger van Thieghem

Cladosporium

cladosporioides (Fres.) Vr.

Cylindrocladium scoparium

Morgan

Epicoccum nigrum Link.

Schol-Schwarz

Fusarium solani (Mart.)

Appel \& Wollenw.

Penicillium sp.

P. citreonigrum Dierckx

P. citrinum Thom

P. corylophylum Dierckx

$P$. decumbens Thom

P. echinulatum Raper \& Thom

P. fellutanum Biourge

P. furcatum Dierckx

P. hirsutum Dierckx

P. implicatum Biourge

P. montanense

Christensen \& Backus

P. olsonii Bain. \& Sartory

P. oxalicum Curie \& Thom

P. purpurogenum Stoll.

P. restrictum Gilman \& Abbott

P. simplicissimum (Oudem.)

Thom

P. spinulosum Thom

$P$. verrucosum Dierckx

$P$. viridicatum Westling

P. waksmanii Zaleski

Diluição Placa

Diluição Placa

\begin{tabular}{llll|llll|llll}
$\mathrm{V}$ & $\mathrm{I}$ & $\mathrm{V}$ & $\mathrm{I}$ & $\mathrm{V}$ & $\mathrm{I}$ & $\mathrm{V}$ & $\mathrm{I}$ & $\mathrm{V}$ & $\mathrm{I}$ & $\mathrm{V}$ & $\mathrm{I}$ \\
\hline & - & - & - & - & - & - & - & - & - & - & - \\
\hline
\end{tabular}

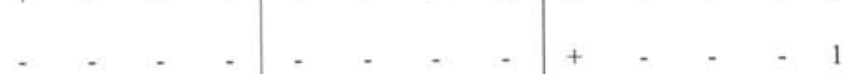

$-\quad-\quad+\quad-\quad-\quad-\quad-\quad-\quad-1$

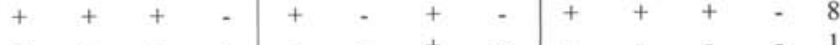

$-++-\frac{-}{-}+-\frac{-}{-}+3$ 


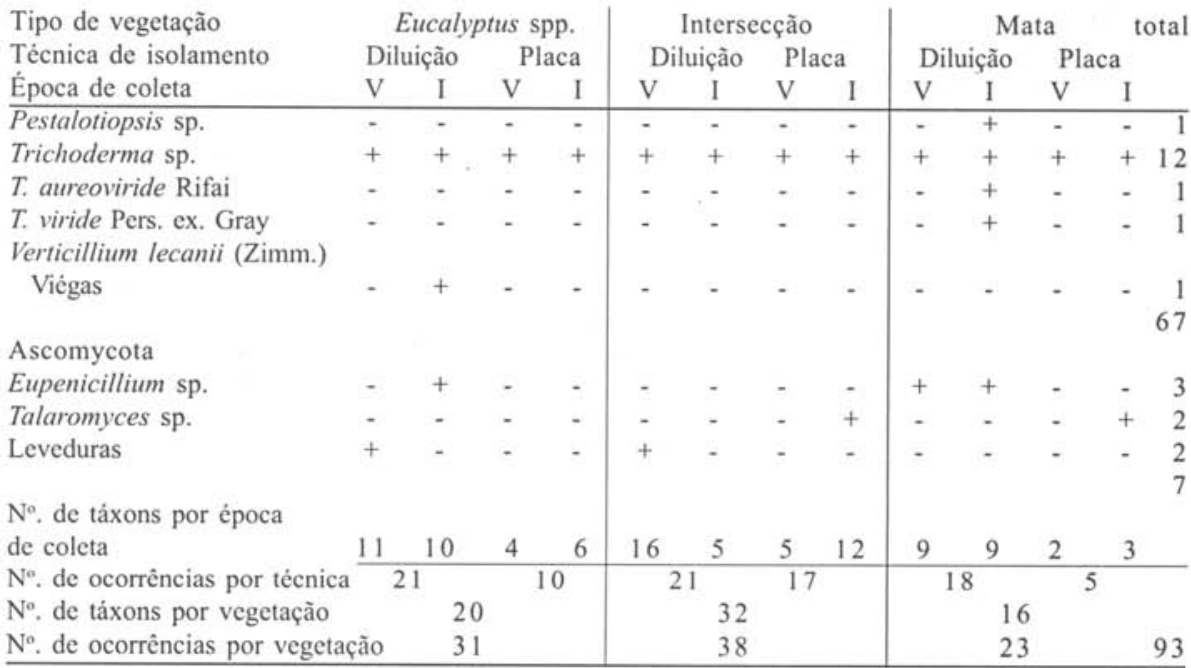

Tabela 3. Médias $(n=5)$ e número total de colônias de fungos isolados pelos métodos de placa de solo (Warcup 1950, modificado) e unidades formadoras de colônias pela diluição em série (Clark 1965), do solo coletado sob três tipos de vegetação na Iltha dos Eucaliptos, Represa do Guarapiranga, São Paulo, SP.

\begin{tabular}{lrrrrrr}
\hline \multirow{2}{*}{ Pontos } & \multicolumn{3}{c}{$\begin{array}{c}\text { No.total de colônias - Placa-de-solo } \\
\text { Eucaliptos }\end{array}$} & \multicolumn{2}{c}{ Interseção } & \multicolumn{2}{c}{ Mata } \\
& verão & inverno & verão & inverno & verão & inverno \\
\hline 1 & 1.112 & 341 & 472 & 652 & 1.040 & 1.176 \\
2 & 534 & 218 & 736 & 433 & 734 & 932 \\
3 & 1.152 & 205 & 524 & 338 & 1.008 & 1.260 \\
4 & 827 & 628 & 556 & 249 & 779 & 547 \\
5 & 656 & 291 & 751 & 175 & 1.100 & 960 \\
Total & 4.281 & 1.683 & 3.039 & 1.847 & 4.661 & 4.875 \\
Média & 856 & 336 & 607 & 369 & 932 & 975 \\
Diluições & \multicolumn{7}{r}{} & $\mathrm{N}^{\circ}$. total de colônias - diluição em série & & \\
$10^{1}$ & 207 & 223 & 403 & 518 & 805 & 852 \\
$10^{2}$ & 290 & 106 & 347 & 163 & 685 & 297 \\
$10^{3}$ & 98 & 21 & 90 & 63 & 235 & 147 \\
$10^{4}$ & 45 & 6 & 32 & 14 & 53 & 93 \\
\hline
\end{tabular}

encontraram alteração no número de colônias, porém verificaram alteração da composição de espécies fúngicas em resposta à adição de extrato da serapilheira de Eucalyptus ao meio de cultura. No presente estudo o possivel efeito inibitório da presença de Eucalyptus sobre os fungos do solo pode ter se manifestado através da diminuição do número de colônias, porém não pela diversidade dos fungos filamentosos. Em estudo paralelo realizado no mesmo local, Wellbaum (1996) isolou fungos filamentosos do folhedo submerso às margens da Represa e também da serapilheira coletada da superficie do solo, encontrando a maior diversidade de táxons na floresta dos Eucalyptus, seguido pela área de interseção e pela mata secundária. Comparações de resultados de isolamento 
Comparações de resultados de isolamento de fungos no campo, com testes in vitro conduzidos no laboratório devem ser efetuadas com o cuidado de levar em consideração possíveis mecanismos de competição, grau de tolerância dos táxons a substâncias antibióticas (do ambiente ou dos próprios fungos), sazonalidade e disponibilidade de nutrientes, entre outros parâmetros, que podem atuar sobre a diversidade dos fungos no solo.

\section{Agradecimentos}

Os autores agradecem aos docentes do Departamento de Botânica e aos técnicos do Laboratório de Ecologia da Universidade de Santo Amaro (UNISA) pelo empréstimo dos equipamentos, e à Dra. Sandra Farto Botelho Trufem pelo auxílio na identificação dos táxons de Mucorales.

\section{Referências bibliográficas}

Almeida, F.F.M. 1981. Mapa Geomorfológico do Estado de São Paulo. Instituto de Pesquisa do Estado de São Paulo, São Paulo, vol.1, 25p.

Antunes, M. F. R.; Ninomiya, A. \& Schoenlein-Crusius, I. H. 1993. Efeitos de queimada sobre a micota de solo na Mata Atlântica na Reserva Biológica do Alto da Serra de Paranapiacaba, SP. Hoehnea 19(2): 1-8.

Attili, D.S. 1994. Isolamento, identificação e ecologia de fungos celulolíticos do solo da Estação Ecológica de Juréia-Itatins, SP. Universidade Estadual Paulista, Rio Claro. Tese de Doutorado.

Barnett, H.L. \& Hunter, B.B. 1972. Illustrated genera of Imperfect Fungi, Burgess Publishing, Mineapolis. Barron, G.L. 1968. The genera of Hyphomycetes from soil. Robert Krieger, New York.

Begovacz, A. 1995. Levantamento florístico preliminar das sub-classes Magnoliidae, Hamamelidae, Caryophyliidae, Dilleniidae, Rosidae (sensu Cronquist, 1981) na Ilha dos Eucaliptos da Represa do Guarapiranga- São Paulo-SP. Universidade de Santo Amaro, São Paulo. Monografia de conclusão de curso de Graduação.

Bettucci, L.; Rodriguez, C. \& Roquebert, M. 1990. Fungal communities of vulcanic ash soils along and altitudinal gradient in Mexico. III. Seasonal variability. Pedobiologia 34: 61-67.

Brown, J.E. \& Zar, J.H. 1984. Field and laboratories methods for general ecology. Brown Publishing, Iowa.

Ceccatto, S.R.; Attili, D.S.; Schoenlein-Crusius, I.H. \& Tauk, S.M. 1986. Aspectos da microbiota fúngica de solo cultivado com Pinus no município de Rio Claro, SP. Revista Brasileira de Ciência do Solo 10: 227230.

Clark, F.E. 1965. Agar-plate method for total microbial count. Pp. 1460-1466. In: C.A. Black; D. Evans; J.L. White; L.E. Ensminger; F.E. Clark \& R.C. Dinauer (Eds.), Methods of soil analysis. Part 2. Chemical and microbiological properties. Madson Inc., New York.

Christensen, M. 1989. A view of fungal ecology. Mycologia 81(1): 1-19.

Crous, P.W.; Knox-Davies, P.S. \& Wingfield, M.J. 1989. A list of Eucalyptus leaf fungi and their potential importance to South African Forestry. Suid-Afrikaanse Bosboutydskrif 149: 17-29.

Della Bruna, E.; Fernandes, B.; Borges, A.C.; Almeida Filho, J \& Barros, N. F. 1989. Efeito do extrato de serapilheira de Eucalyptus sobre o crescimento microbiano. Pesquisa Agropecuária Brasileira 24(12): 1523-1528.

Della Bruna, E.; Borges, A.C.; Fernandes, B.; Barros, N.F. \& Muchovej, R.M.C. 1991. Atividade da microbiota de solos adicionados de serapilheira de eucalipto e de nutrientes. Revista Brasileira de Ciência do Solo 15: $15-20$.

Difco Manual, 1972. 9 ed., Difco Manual, Difco Laboratories, Michigan .

Dix, N.J. \& Webster, J. 1995. Fungal Ecology. Chapman \& Hall, London.

Domsch, K.H.; Gams, W. \& Anderson, T.H. 1980. Compendium of soil fungi. Academic Press, New York, 2v. Emplasa. 1981. Levantamento Aerofotogramétrico. Folha Jardim Iporanga. São Paulo, Índice de nomenclatura SF-23-4-VI-2-50. 
Garlipp, A. B. 1995. Isolamento e identificação de fungos filamentosos do solo do Banhado Grande, na Estação Ecológica de Juréia-Itatins, SP. Universidade Estadual Paulista, Rio Claro. Dissertação de Mestrado.

Garrett, S.D. 1963. Soil fungi and fertility. Pergamon Press, Oxford.

Lima, W.P. 1993. Impacto ambiental do Eucalipto. EDUSP, São Paulo.

Martin, W. 1961. Introduction to soil microbiology. Blackwell, London.

Mc Lean, R.C. \& Cook, W.R.I. 1968. Practical field ecology: a guide for the Botany Department of Universities, Colleges and Schools. George Allen, London.

Ninomiya, A.; Antunes, M.F.R. \& Schoenlein-Crusius, I.H. 1993. Fungi from soil affected by birds in the Parque Estadual das Fontes do Ipiranga, São Paulo State, Brazil. Revista de Microbiologia 24: 49-53.

Pitt, J. I. 1979. The genus Penicillium and its teleomorphic states Eupenicillium and Talaromyces. Academic Press, New York.

Poggiani, F. 1995. Aspectos ambientais da exploração de florestas naturais de plantação de Eucaliptos. Publicação da Sociedade Brasileira de Silvicultura 62: 10-15.

Raij, B. \& Zullo, M.A.T. 1977. Métodos de Análises de Solo. Instituto Agronômico de Campinas, Campinas, circular n $63,16 \mathrm{p}$.

Riffai, M.A. 1969. A revision of the genus Trichoderma. Mycological Papers 116: 1-56.

Rocha, A.A. 1976. A Limnologia, os aspectos ecológicos, sanitários e a macrofauna bentônica da Represa do Guarapiranga na região metropolitana de São Paulo. Universidade de São Paulo, São Paulo. Tese de Doutorado.

Salomão, C.C. 1993. Vilão ou Herói? Sociedade Brasileira de Silvicultura 50: 22-23.

Schoenlein-Crusius, I. H. 1993. Sucessão fúngica em folhas de Alchornea triplinervia (Spreng.) M. Arg. em ambientes aquático e terrestre, na Mata Atlântica, Reserva Biológica do Alto da Serra de Paranapiacaba, Santo André,SP. Universidade Estadual Paulista, Rio Claro. Tese de Doutorado.

Sutton, B.C. \& Hodges, J.C.S. 1975. Eucalyptus microfungi: two new Hyphomycete genera from Brasil. Nova Hedwigia 26: 257-533.

Sutton, B.C. \& Hodges, J.C.S. 1976. Eucalyptus microfungi: some setose Hyphomycetes with phialides. Nova Hedwigia 27: 343-352.

Warcup, J. H. 1950. The soil plate method for isolations of fungi from soil. Nature 166: 117-118.

Wellbaum, C. 1996. Estudo preliminar dos fungos filamentosos em folhas do ambiente terrestre e aquático da Ilha dos Eucaliptos, Represa do Guarapiranga, município de São Paulo, SP. Universidade de Santo Amaro, São Paulo. Monografia de conclusão de curso de Graduação. 